\title{
Nematodos en caña de azúcar, Virú - La Libertad
}

Nematodes in sugarcane, Virú - La Libertad

${ }^{1}$ Charles Frank Saldaña Chafloque ${ }^{a}$

\section{RESUMEN}

La producción de "caña de azúcar" Saccharum officinarum L. en el Perú se concentra en la costa del país, siendo la principal zona productora, sus derivados son etanol, melaza, vinaza entre otros. Los géneros de nematodos fitoparásitos hallados en las muestras de suelo y raíz del cultivo de "caña de azúcar", sembradas en la provincia de Virú, departamento de La Libertad son, Meloidogyne sp, Pratylenchus sp, Helicotylenchus sp, Tylenchorhynchus sp, Criconemoides sp, Hemicycliophora sp, Trichodorus sp, Tylenchus sp, Rotylenchus sp, Aphelenchus sp, Scutellonema sp. Asimismo, las especies de nematodos antes mencionadas provocan las reducciones en la producción de caña de azúcar de un 30 a 50\%, en condiciones de invernadero y campo. Por ello se establece los siguientes controles para el manejo de los nematodos, tales como el Control varietal, uso de variedades resistentes y tolerantes, Control químico, nemotóxicos, Control cultural: Enmiendas orgánicas, Fertilización completa y control de malezas y Control biológico: Uso de bacteriófagos, hongos parásitos. La continua demanda por azúcar, obliga a incorporar a la agricultura zonas arenosas y de bajo nivel productivo; nicho aprovechado por nematodos Fitopatógenos, que se proliferan rápidamente ante una débil fauna antagónica existente.

Palabras clave: nematodo, caña de azúcar, métodos de control

\begin{abstract}
The production of "sugarcane" Saccharum officinarum L. in Peru is concentrated in the coast of the country, being the main producing area, its derivatives are ethanol, molasses, vinasse among others. The genero of phytoparasitic nematodes found in the soil and root samples of the "sugarcane" crop, planted in the province of Virú, La Libertad department, are Meloidogyne sp, Pratylenchus sp, Helicotylenchus sp, Tylenchorhynchus sp, Criconemoides sp, Hemicycliophora sp. Trichodorus sp, Tylenchus sp, Rotylenchus sp, Aphelenchus sp, Scutellonema sp. Likewise, the aforementioned nematode species cause reductions in the production of sugar cane from 30 to $50 \%$, under greenhouse and field conditions. Therefore, the following controls are established for the management of nematodes, such as varietal control use of resistant and tolerant varieties; chemical control, nemotoxics; cultural control, organic amendments, complete fertilization and weed control and biological control, use of bacteriophages, parasitic.
\end{abstract}


The continuous demand for sugar, forces to incorporate to agriculture sandy areas and of low productive level; niche harnessed by phytopathogenic nematodes, which proliferate rapidly in the presence of a weak existing antagonistic fauna.

keyword: nematode, sugar cane, control methods

${ }^{1}$ Universidad Nacional Autónoma de Tayacaja Daniel Hernandez Morillo, Huancavelica - Perú. ${ }^{\text {a } B i o ́ l o g o . ~}$ 


\section{INTRODUCCIÓN}

\section{Perspectivas del cultivo de caña en el Perú}

La producción de caña de azúcar en el Perú se concentra en la costa del país, siendo la principal zona productora. Tiene un gran potencial de desarrollo agroindustrial, derivado de la azúcar, etanol y otros productos derivados de su producción. Al cierre del 2018 , ascendieron a 160,000 las hectáreas de caña de azúcar cultivadas en el país, que representaron un $17 \%$ más que al cierre del 2017 (136,000). Esta es la mayor superficie registrada en los últimos 30 años en el Perú. Los departamentos que concentran la mayor superficie cosechada son La Libertad con el $45.7 \%$ y Lambayeque con el $31.7 \%$, ambos concentran el $77.4 \%$ de la superficie cosechada nacional. Los demás departamentos concentran el $22.6 \%$, esto es Lima con $14.9 \%$, Ancash con $7.0 \%$ y Arequipa con $0.7 \%$ respectivamente. (Ministerio de Agricultura, 2019).

En Perú, el cultivo de caña de azúcar representa una importante actividad agrícola, actualmente este cultivo se viene sembrando con sistema de riego por goteo, como en los proyectos de Maple Etanol o Comisa que aportarán cerca de 25000 hectáreas de caña de azúcar con este sistema en la costa norte de Perú. La Empresa Agroindustrial Laredo en Trujillo, La Libertad, del grupo Manuelita, que comprenden 3000 hectáreas y Agrícola La Chira en Piura, del grupo Romero, con 7000 hectáreas. Con este sistema de riego por goteo el país posiblemente desplazaría a Sudáfrica como primer productor mundial de caña de azúcar cultivada con riego por goteo (Municipalidad Provincial de Piura, 2010).

La empresa Agrícola La Chira, es el único productor de alcohol carburante a partir de caña de azúcar, cuenta con una capacidad de producción de 350000 L/día; esta planta entró en producción a fines del tercer trimestre del año 2 009. En este mismo valle, se ubican dos proyectos para la producción de etanol: uno es la empresa MAPLE y el otro la empresa COMISA, con áreas de cultivo de 10000 y 15000 has respectivamente, ambos proyectos están orientados a la exportación (Ministerio de Agricultura, 2013).

\section{NEMATODOS EN CAÑNAE AZÚCAR}

\section{Especies de nematodos en el cultivo de caña de azúcar, Virú. La Libertad}

Los nematodos fitoparásitos hallados en las muestras de suelo y raíz del cultivo de "caña de azúcar" Saccharumm officinarum L., sembradas en la provincia de Virú, departamento de La Libertad son, Meloidogyne sp, Pratylenchus sp, Helicotylenchus sp, Tylenchorhynchus sp, Criconemoides sp, Hemicycliophora $s p$, Trichodorus sp, Tylenchus sp, Rotylenchus sp, Aphelenchus sp, Scutellonema sp. Ferreyros, 1983; Martowo, 1987, Suarez, 2000. 


\section{Signos y Síntomas causados por nematodos}

Los síntomas producidos por Meloidogyne son enanismo y amarillamiento de las hojas. Se presentan como deficiencia de agua en las horas de mayor calor, ya que las raíces son dañadas por el agallamiento en las mismas las cuales son manifestaciones externas, $\mathrm{y}$ que internamente se presentan desde el momento de la penetración de las larvas juveniles del segundo estadio. Las cuales provocan una serie de cambios en los tejidos radicales (Taylor y Sasser, 1983).

\section{Estimación de pérdidas por nematodos en caña de azúcar}

Existen 310 especies de nematodos endoparásitos y ectoparásitos, incluidas en 48 géneros registrados en el cultivo de caña en el mundo (Cadet y Spaull, 2005). Se ha comprobado que los nematodos fitoparásitos necesitan, para completar su ciclo de vida, una relación muy cercana con su hospedero el cual le provee alimento y condiciones favorables para su desarrollo. Estas dependencias a los cambios indican que la relación entre planta y patógeno es predecible puesto que la presión de cambio en la vegetación hospedera afecta a los organismos vivos que dependen de ella. (Tovar, 2011, Salazar y Guzmán 2013).

Especies de nemátodos que atacan al cultivo de la caña de azúcar como Meloidogyne incognita, M. javanica y Pratylenchus zeae Graham, (Novaretti et al., 2010). Asimismo, las especies de nematodos antes mencionadas provocan las reducciones en la producción de caña de azúcar de un 30 a 50\%, en condiciones de invernadero y campo (Dinardo Miranda et al., 2008).

Los nematodos Fitoparásitos, al ser los responsables directos de dañar el sistema radicular del cultivo de la caña de azúcar, estos se ven limitados en la absorción de agua y nutrientes que se encuentran en el suelo y por tanto no pueden suministrar las cantidades suficientes que la planta en desarrollo está demandando y como respuesta inmediata de la planta al no tener suficiente nutrimentos, esta detiene su crecimiento o lo hace muy lentamente y por tanto no llega a acumular la cantidad necesaria de biomasa (hojas, raíces y lo más importante no se desarrolla bien los tallos molibles), resultando con bajo rendimiento de campo (ton/ha).

Según Andres, M. 2002, las pérdidas de cosecha anuales estimadas debidas a nematodos fitoparásitos en la producción agrícola mundial se aproxima al $11 \%$ y en términos absolutos las pérdidas económicas anuales se calculan en torno a los 100 billones de dólares.

\section{Daños de los nematodos en la caña de azúcar}

Los nematodos son causantes de los siguientes daños en el cultivo de caña de azúcar, de la provincia de Virú: reducción de la masa radicular, lesiones en el sistema radicular, muerte de células de la raíz o modificación de las mismas por la alimentación de los fitonemátodos, 
apertura de entrada a otros patógenos, Reducción de la longevidad de los cañaverales, como consecuencia de los daños causados por los nematodos tenemos la reducción de la productividad (bajo rendimiento de campo), entre otros (Taylor y Sasser, 1983).

\section{Hábitat de Meloidogyne spp}

Todas las especies de Meloidogyne se favorecen de texturas de suelo gruesas, suelos arenosos y muy pocas veces se encuentra a este género en suelos de textura fina con altos porcentajes de porosidad y arcillosos (Bridge y Starr, 2007). Según Jaraba, Lozano y Espinosa (2007) en su investigación presentaron la relación de especie con los factores edafológicos en los cuales $M$. incognita se encontró en suelos con pH de 4,9 a 6,4; MO de 1,2 a 2,5\%; contenidos de arenas que oscilan entre 50 y $86 \%$, arcillas de $3 \%$ a $17 \%$ y limos de 11 a $38 \%$.

La combinación de especies predominantes ( $M$. incognita y $M$. arenaria) se hallarón en suelos con $\mathrm{pH}$ de 5,0 a 6,0; MO de 1,5 a 2,5\%; contenidos de arenas que oscilan de 51 a $60 \%$, arcillas del 9 a 14\% y limos del 31 a 33\%. La asociación de las tres especies ( $M$. incognita, M. javanica y M. arenaria) se encontró en suelos con pH de 4,9 a 6,4; MO de 1,2 a 1,5\%; contenidos de arenas que oscilan de 53 a $87 \%$, arcillas de 3 a $17 \%$ y limos de 11 a $37 \%$ (Jaraba et al, 2007).

Líneas arriba, muestra la capacidad la capacidad del nematodo de adaptarse a los diferentes tipos y condiciones de suelos. El rango adecuado para su reproducción y sobrevivencia de $\mathrm{pH}$ de 4 a 8 . Así como la asociación de las poblaciones de Meloidogyne a suelos con contenidos medios y altos de arenas más comúnmente que suelos altos en arcillas. Favoreciendo a su presencia el contenido de arena y limitando las poblaciones el $\mathrm{pH}$, la materia orgánica y la conductibilidad eléctrica (Jaraba et al, 2007).

\section{Ciclo de Vida de Meloidogyne ssp}

La reproducción ocurre cuando el segundo estadio larval infectivo (J2) penetra en las raíces, justamente sobre la caliptra (punta de la raíz). Se movilizan entre las células no diferenciadas de la raíz y colocan sus cabezas en el cilindro central de desarrollo, cerca de la región de elongación celular, y sus cuerpos en la corteza. Con sus estiletes perforan las paredes de las células e inyectan secreciones de sus glándulas esofágicas ( Tay lor y S a s ser, 1983 ).

Su acción provoca la aparición de nódulos en las plantas. En hospederos susceptibles por medio de su estilete perforan las células en el cilindro vascular, incrementando así la proporción de la división celular, originándose células gigantes, llamadas sincitios. La respuesta de la planta es el engrosamiento de la raíz formando agallas visibles (Taylor y Sasser, 1983; Cepeda, 1996). 


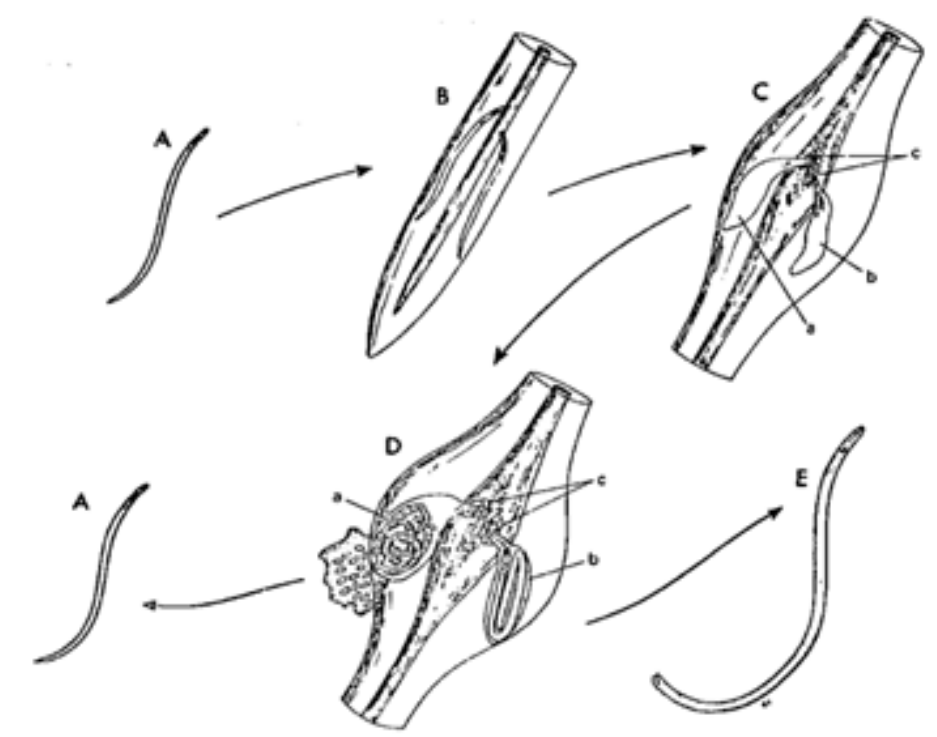

Figura 1. Ciclo esquemático de vida de una especie de Meloidogyne.

El ciclo de vida de Meloidogyne ssp, comprende las siguientes etapas: A) Segundo estadio larval pre-parasítico. B) Dos larvas que han penetrado en una raíz, haciéndose sedentarias y comenzando su alimentación. C) Inicio de la formación de agallas, desarrollo de larvas $(\mathrm{a}, \mathrm{b}) \mathrm{y}$ células gigantes (c). D) Agalla con una hembra madura y su masa de huevos (a), macho de la metamorfosis (b), y células gigantes (c). E) Macho libre en el suelo (Taylor y Sasser, 1983).

\section{MANEJO DE NEMATODOS EN CAÑA DE AZÚCAR}

Según Andrés, M. 2002, el control de nematos debería ser preventivo, por lo tanto, la prevención frente a la introducción de nematodos en áreas donde no se encontraban anteriormente debería ser la primera medida de control. Antes de decidir qué tipo de técnicas de control son las más adecuadas en cada caso, es necesario caracterizar el problema, determinar los nematodos patógenos y conocer su densidad, biología e interacción específica con el cultivo. Para ello se establece los siguientes controles para el manejo de los nematodos: Control varietal: uso de variedades resistentes y tolerantes, Control químico: nemotóxicos, Control cultural: Enmiendas orgánicas, Fertilización completa y control de malezas y Control biológico: Uso de bacteriófagos, hongos entre otros.

\section{Control Varietal}

La obtención de variedades resistentes se lleva por medio de la hibridación de plantas susceptibles con plantas resistentes, a través de los cruzamientos de individuos, uno es una variedad comercial que es necesario introducirle la resistencia de otro individuo. La primera 
generación que se obtiene los hibrido, los cuales se van a cruzar con el progenitor para solo fijar las características deseadas, que en este caso es resistencia (Cepeda, 1996).

\section{Control químico}

El uso de nematicidas es una forma práctica para controlar al nematodo de los nódulos radiculares en cultivos. Entre los nematicidas recomendados se encuentran bromuro de metilo, metam sodio, oxamyl. Aunque estos materiales son efectivos presentan un riesgo latente de seguridad y daños al medio ambiente (Brust et al, 2003, Appleman y Hanmer, 2003). Además, cabe mencionar que es muy importante el desarrollo de métodos de control no selectivos y más económicos como los métodos de biocontrol (Noling, 2005).

\section{Control Cultural}

La presencia de métodos de control dirigidos a disminuir las poblaciones del patógeno en un área, en el cultivo de caña de azúcar. A estas prácticas se les conoce como métodos de control cultural. Entre las prácticas culturales más utilizadas son la rotación de cultivos, barbecho, inundación, solarización, aportes de las enmiendas orgánicas contra los nematodos: Vigorizan al cultivo, colchón de agua y nutrientes, mejoran estructura suelo, mayor exploración de raíces, estimulación de microorganismos antagónicos, liberan sustancias toxicas, Fertilización completa y oportuna: Incluyendo fosforo, potasio y calcio, fertilización con micro-elementos Inclusive hormonas: Ac. Giberelico. (Santiago, 2006, UF/IFAS, 2008)

\section{Control de malezas}

Una estrategia para el manejo de nematodos consiste en evitar que el microorganismo continúe con su ciclo de vida al eliminar plantas hospederas. Por tal motivo el propósito de eliminar hospederos es para romper el ciclo, siendo necesario poner especial atención a las especies de malezas que fungen como hospederos (Chenopodiaceas, Solanáceas, Amarantáceas, Compuestas, Gramíneas).

\section{Control biológico}

Entre los principales controladores biológicos de nematodos formadores de agallas se encuentran las bacterias y los hongos, detallándose a continuación Hongos: Paecelomyces lilacinus, Verticillium lecanii, Verticillium chlamidosporiu, Catenaria vermicola, Arthrobotrys oligospora, Dactylella oviparasitica, Dactylaria sp y Cylindrocarpon destructans. Bacterias: Pasteuria penetrans, Streptomyces avermitilus, Burkholderia tropicalis y Bacillus thuringiensis Ciancio, A.; 1999., Rojas, M. 1999, Giannakou, I. O. 2002, Jonathan, E. 2000, Schenck, S. 2004, 
Holland, R. J. 2001. Holland, R. J. 1999, López, J. A. 2004, Dong, L. Q. 2006. Kerry, B. R. 2001, Hernández, M. A. 2008, Gullino, M. 2003, López, J. A. 2004.

\section{CONSIDERACIONES FINALES}

La continua demanda por azúcar, obliga a incorporar a la agricultura zonas arenosas y de bajo nivel productivo; nicho aprovechado por nematodos Fitopatógenos, que proliferan rápidamente ante una débil fauna antagónica existente.

Existe una comunidad de nematodos que perjudican de forma indirecta al cultivo de caña de azúcar, disminuyendo sus producciones.

El control de los nematodos en caña de azúcar está apuntando a la utilización de variedades resistentes y tolerantes, actualmente se usa métodos de control contra los nematodos.

Todas las acciones que ayuden a la vigorización del cultivo, ya sea enmiendas orgánicas, buena fertilización, control de malezas, aminoran la severidad del daño por nematodos.

Realizar estudios exploratorios mediante el muestreo de raíz o suelo en cada una de las áreas de influencia de los ingenios para verificar este estudio.

Hacer estudios mediante el establecimiento de parcelas experimentales donde se evalúen diversas alternativas de control que ayuden a minimizar el impacto que esta plaga provoca al sistema radicular de la caña de azúcar.

Capacitar a técnicos y productores para realizar estudios de muestreo radicular y el envío de muestra al laboratorio para su análisis correspondiente.

\section{REFERENCIAS BIBLIOGRÁFICAS}

Andres, M. 2002. Estrategias En El Control Y Manejo De Nematodos Fitoparásitos. Ciencias Y Ambiente - Ccma - Csic Http://Digital.Csic.Es/Bitstream/1 0261/128310/1/Estrategias $\% 20$ en $\% 20$ el $\% 20$ control392\% $28 \mathrm{~m} \%$ C2\%Aaf\%20andr\%C3\%A9s\%29. Pdf

Appleman, L. And D. Hanner. 2003. Screenming For Root-Knot Nematode (Meloidogyne Hapla) Using Lettuce. Uw-L Journal Of Undergraduate.

Bridge, J., Starr, J. (2007).Plant Nematodes Of Agricultural Importance. Academic Press. Ee.Uu. 152pp.

Brust, E.G, W.D. Scout And J.M. Ferris. 2003. Root-Knot Nemtode Control In Melons. Departament Of Entomology. Disponible En: Http://72.14.205.104/ Search? $\mathrm{Q}=$ Cache

Cadet, P., And V. W. Spaull. 2005. Nematodes Parasites Of Sugarcane. In: Luc, M., R. A. Sikora, And J. Bridge (Eds). Plant Parasitic Nematodes In 
Subtropical And Tropical

Agriculture. Cab International, London. Pp: 645-674.

Cepeda S., M. 1996. Nematología Agrícola. Editorial Trillas, S.A. De C.V. México, D. F. Pp 132188.

Ciancio, A.; Carbonell, E. Y Crozzoli, R. "Ecología Y Biodiversidad De Pasteuria Spp., Antagonistas Naturales De Nematodos Fitoparasíticos". Fitopatología Venezolana, 11(1): 1-9, 1999.

Dinardo-Miranda, L. L.; Pivetta P. J.; Fracasso, V. J., 2008. Influência

Da Época De Aplicação De Nematicidas Em Soqueiras Sobre As Populações De Nematóides E A Produtividade Da Cana-De-Açúcar. Bragantia, Campinas, V.67, N.1, P.179-190.

Dong, L. Q. Y Zhang, K. Q.: "Microbial Control Of Plant-Parasitic Nematodes: A Five Party Interaction". Plant Soil, 288: 3145, 2006.

Ferreyros C., P. 1983. Cultivo De Marigold (Tagetes Erecta L). Folleto Compañía Alimentos Deshidratados S.A. (Alidesa) Perú. 19p.

Giannakou, I. O.; Gowen, S. R. Y Davies, K. G.: "Aspects On The Attachment Of Pasteuria Penetrans On Root-Knot

Nematodes". Russian Journal of Nematology, 10: 25-31, 2002.
Biologici E Prodotti Di Origine Naturale Per La Difesa Dai Parassiti Terricoli". Informatore Fitopatologico, 10: 51-57, 2003. Hernández, M. A. Y Hidalgo-Díaz, L.: "Klamic: Bionematicida Agrícola Producido A Partir Del $\mathrm{H}$ o n g o Pochon $i$ a Chlamydosporia Var. Catenulata". Rev. Protección Veg., 23(2): 131-134, 2008.

Holland, R. J.: "Paecil", 2001. Http://Www.Ticorp.Com.Au/Ar ticle1.Htm. (Consultado Mayo 2004).

Holland, R. J.; Williams, K. L. Y Khan, A.: "Infections of The Interaction Of Paecylomices Lilacinus With Meloidoyne Incognita". Nematology, 1: 131139, 1999.

Https:/Www.Intagri.Com/Articulos/Fito s a n i d a d / Co n trol-De Nematodos-Desde-UnaPerspectiva-Integral.

Intagri. 2017. Control De Nematodos Desde Una Perspectiva Integral. Serie Fitosanidad Núm. 91. Artículos Técnicos De Intagri. México. 5 P. Disponible En:

Jaraba, J. D., Lozano, Z. Y Espinosa, M. (2007). Nematodos Agalladores Asociados Al Cultivo De Papaya (Carica Papaya L.) En E1 Departamento De Cordoba, Colombia. Agronomía Colombiana. 124-130.

Gullino, M. Y Benuzzi, M.: "Mezzi 
Jonathan, E I.; Arulmozhiyan, R.; Muthusamy, S. Y Manuel, W. W.: "Field Application Of Paecilomyces Lilacinus For The Control Ofmeloidogyne Incognita On Betelvine, Piper Betle". Nematol. Medit., 28(2): 131-133, 2000.

Kerry, B. R.: "Exploitation Of The Nematophagous Fungus Verticillium Chlamydosporium Goddard For The Biological Control Of Root-Knot Nematodes (Meloidogyne Spp.)". En: Fungi As Biocontrol Agents:

Progress, Problems And Potential (Butt, T. M.; Jackson, C Y. Magan, N, $E d s)$. Cabi International, Wallingford. Chapter 5: 155168, 2001.

López, J. A.: "Control Biológico De Nematodos Parásitos De Plantas". En: Control Biológico De Plagas Agrícolas (Caraballo, M. Y Guaharay, F.Eds): 185-200. Serie Técnica, Manual Técnico Centro Agronómico Tropical De Investigación Y Enseñanza, 53, 2004.

López, J. A.: "Control Biológico De Nematodos Parásitos De Plantas". En: Control Biológico De Plagas Agrícolas (Caraballo, M. Y Guaharay, F.Eds): 185-200. Serie Técnica, Manual Técnico Centro Agronómico Tropical De Investigación $Y$ Enseñanza, 53,
2004.

Martowo, B. Rohaha D. 1987. The Effect Of Intercropping Of Pepper (Capsicum Annum L.) With Some Vegetable Crops On Pepper Field And Disease Incidence Caused By Meloidogyne Spp. Boletin Penelitian Hortikultura 15(4):55-59.

Ministerio De Agricultura. 2013.

Dirección General De Competitividad Agraria. Primera Edición. Perú. 11-30p.

Ministerio De Agricultura. 2019. Boletín Estadístico Mensual. Lima. Perú. Dirección General De Seguimiento Y Evaluación De Políticas Dgesep. Dirección De Estadística Agraria Dea. Perú. $147 \mathrm{p}$.

Municipalidad Provincial De Piura. 2010.

Plan De Desarrollo Urbano Metropolitano De Piura. Perú. 1$228 p$.

Noling, J.W.2005 Nematode Management In Cucurbits (Cucumber, Melons, Squash). Entomology And Nmatology De partamnet. Florida Cooperative Extension Service. Institute Of Food And Agricultural Sciencies. University Of Florida. Eny-025. P104.

Novaretti, W. R. T. ; Carderan, J. O., 2010. Efeito Do Controle De Nematoides $\mathrm{Na}$ Adubaçao 
Fosfatada Da Cana-De-Açúcar Cultivada Na Regiao Oeste Do Estado De Sao Paulo. Revista Stab. Vol. 28 No.3. P 32-37.

Peña, M. 1997. Propagación In Vitro De La Caña De Azúcar. Tesis Ing. Agr. Zamorano. Honduras. 39p.

Rojas, M. T. Y Marban-Mendoza, N.: "Pasteuria Penetrans. Adherencia Y Parasitismo En Meloidogyne Incognita Y Meloidogyne Arabicida". Nematropica, 29: 233-240, 1999.

Salazar W.A Y T De J Guzmán Hernández. 2013. Nematodos Fitoparásitos Asociados Al Tomate En La Zona Occidental De Nicaragua. Agronomía Mesoamericana 24(1):27-36. Issn: 1021- 7444.

Santiago G., J.C. 2006. Manejo Integrado de Nematodos Fitoparásitos Cosmopolitas (Gemmar) En El Cultivo de Plátano (En Línea). Universidad de Puerto Rico. Recinto Universitario De Magaguuez. Disponible En: Http://Grad.Uprm.Edu/Tesis/Sa ntiagogonzales.Pdf

Schenck, S.: "Control Of Nematodes In Tomato With Paecilomyces Lilacinus Strain 251". Hawaii Agriculture Research Center, Vegetable Report, (5): 5, 2004.

Suárez, Z. 2000. Nemátodos Asociados a los Frutales y su Control. Ceniap. Maracay. Disponible
Http://Fonaiap.gob.ve/pública/d ivulga/fd59/Nemato

Taylor, A., Sasser, J. (1983). Biología, Identificación Y Control De Los Nematodos De Nódulo De La Raíz. Departamento De Fitopatología De La Universidad Del Estado De Carolina Del Norte.

Tovar C.C.A. 2011 Efecto De La Modificación D e L a Temperatura Del Suelo Sobre La Densidad Y Riqueza De Grupos Funcionales De Nematodos En Bosque Altoandino (Cuenca Río Blanco, Cundinamarca).Trabajo De Grado. Presentado Como Requisito Parcial Para Optar Al Título De Microbióloga Industrial. Pontificia Universidad Javeriana Facultad de Ciencias Carrerade Microbiología Industrial, Bogotá, D. C. 50 Pp Consultado El 15 De Enero Del 2016 Y D i s p o n i b l e En : Http://Repository.Javeriana.Edu .Co/Bitstream/10554/8815/1/Te sis762.Pdf

Uf/Ifas (University Of Florida And Institute Of Food And Agricultural Sciences), 2008. Management Integrated Of Nematodes. ( Uf/Ifas). D i s p o n ible E n : Http://Traslate.Google.Com.Mx /Traslate $? \mathrm{Hl}=\mathrm{Es} \& \mathrm{Sl}=\mathrm{Es} \& \mathrm{U}=\mathrm{Ht}$ tp://Edis.Ifas.Efl.Edu/Ng032\&S 
$\mathrm{a}=\mathrm{X} \& \mathrm{Oi}=$ Traslate $\&$ Resnum $=2$

$\& \mathrm{Ct}=$ Result\&Prev $=/$ Search $\% 3 \mathrm{f}$

$\mathrm{q} \% 3$ dmanagement $\% 2$ bintegrate

$\mathrm{d} \% 2$ bnematodes $\% 26 \mathrm{hl} \% 3 \mathrm{des}$.

\section{CORRESPONDENCIA}

M.Sc. Charles Frank Saldaña Chafloque

charles.saldana.chafloque@gmail.com 